\title{
Dabigatran: Review of Pharmacology and Management of Bleeding Complications of This Novel Oral Anticoagulant
}

\author{
Michael Ganetsky • Kavita M. Babu • \\ Steven D. Salhanick - Robert S. Brown • \\ Edward W. Boyer \\ Published online: 2 September 2011 \\ (C) American College of Medical Toxicology 2011
}

\begin{abstract}
Dabigatran (Pradaxa) is a competitive direct thrombin inhibitor approved by the US FDA for prevention of embolic stroke in patients with nonvalvular atrial fibrillation. Dabigatran has a pharmacokinetic profile that produces predictable anticoagulation responses, does not undergo CYP 450 metabolism, has few drug-drug and drug-food interactions, and does not require frequent laboratory monitoring of clotting parameters. Clinicians are rapidly prescribing this agent as a replacement for warfarin therapy. However, no therapeutic agent has been accepted to reliably reverse the hemorrhagic complications of dabigatran. As of yet, there is no solid evidence to guide management of bleeding complications; management should start with local control of bleeding when possible and transfusion of pRBCs if needed. Transfusion of FFP would not be expected to help control bleeding. Limited and mixed data exist for transfusion of factor VIIa and
\end{abstract}

\footnotetext{
M. Ganetsky $(\bowtie) \cdot$ S. D. Salhanick

Department of Emergency Medicine,

Beth Israel Deaconess Medical Center,

One Deaconess Rd, WCC-2,

Boston, MA 02215, USA

e-mail: mganetsk@bidmc.harvard.edu

K. M. Babu

Division of Medical Toxicology. Department of Emergency

Medicine, The Alpert Medical School/Brown University,

Providence, RI, USA

R. S. Brown

Department of Internal Medicine, Renal Division,

Beth Israel Deaconess Medical Center,

Boston, MA, USA

E. W. Boyer

Department of Emergency Medicine,

UMass Memorial Medical Center,

Worcester, MA, USA
}

prothrombin complex concentrates; these therapies should be considered as well as dialysis, which will increase elimination in patients with life-threatening or closed-space bleeding due to dabigatran. We present an article that reviews the pharmacokinetics, clinical trial literature, and consensus guidelines regarding this novel oral anticoagulant.

Keywords Dabigatran P Pradaxa $\cdot$ Direct thrombin inhibitors $\cdot$ Hemodialysis

\section{Introduction}

In 2010, the United States Food and Drug Administration approved dabigatran, the first new oral anticoagulant to be approved in the United States of America in 50 years. Dabigatran is indicated for the prevention of embolic stroke in patients with nonvalvular atrial fibrillation. The use of dabigatran as an alternative to warfarin received a class I recommendation from the American College of Cardiology and the American Heart Association in February 2011 [1]. The United States anticoagulant market is expected to grow to over US $\$ 9$ billion in 2014 from US $\$ 6$ billion in 2008; direct thrombin inhibitors and factor Xa inhibitors combined will comprise over half this market [2].

Dabigatran differs from conventional warfarin-based anticoagulation therapy in two important ways. The stable hematologic response to dabigatran administration makes frequent laboratory monitoring of clotting parameters unnecessary, an appealing feature for many patients and providers. Of greater concern, however, is that there is little experience with dabigatran-induced bleeding, and no therapeutic agent reliably reverses hemorrhagic complications of dabigatran therapy.

We reviewed current English language literature on dabigatran pharmacokinetics, pharmacology and monitoring, 
the clinical trials literature, societal consensus guidelines, review articles, and the pharmaceutical's package insert. We selected sources that were frequently referenced and provided thorough content to synthesize basic principles and explore controversial areas; as this review does not focus on efficacy, the strength of the clinical trials is not discussed. In this article, we present the pharmacology and pharmacokinetics of dabigatran, the clinical trials that led to its approval, pitfalls in the assessment of intensity of anticoagulation, and potential issues and strategies in the management of bleeding complications.

\section{Pharmacology}

Dabigatran is a reversible, potent, competitive direct thrombin inhibitor (Fig. 1) [3]. Unlike heparin, which can only bind free thrombin, dabigatran is capable of binding and inhibiting both free and clot-bound thrombin [4]. Understanding the role of thrombin in coagulation is central to a discussion of the mechanism of action of dabigatran and other anticoagulants.

Endothelial injury results in the exposure of tissue factor which interacts with activated factor VII to initiate the coagulation cascade. Thrombin, a serine protease, enables thrombus formation by activating factors V, VIII, and XI and catalyzing the conversion of fibrinogen to fibrin [5]. Additionally, thrombin causes platelet aggregation by activating protease-activated receptors on the platelet surface $[6,7]$.

Thrombin also initiates fibrinolytic events, as the thrombinthrombomodulin complex activates protein $\mathrm{C}$, which subsequently inactivates factors Va and VIIIa. Antithrombin III, a serine protease inhibitor, inactivates factors Xa, IXa, XIIa, and IIa. The anticoagulative effects of antithrombin III are accelerated hundreds to thousands of times in the presence of unfractionated and low-molecular weight heparins $[8,9]$.

Structurally, thrombin contains three major drug binding domains: the active (or serine protease catalytic) site and two exosites (1 and 2). Exosite 1 binds fibrin, while exosite 2 binds heparin [10]. Heparin-bound thrombin can then bind fibrin or antithrombin. Once heparin-bound thrombin is joined to fibrin, both exosites are occupied, and the complex is relatively protected from the effects of antithrombin. As a result, heparin has little effect on fibrinbound thrombin [11-13].

In contrast, direct thrombin inhibitors, such as dabigatran, can inhibit free, fibrin-bound, and clot-bound thrombin. Bivalent direct thrombin inhibitors, such as hirudin and bivalirudin, bind both the active site and exosite 1, while univalent direct thrombin inhibitors, like dabigatran, bind only the active site (Fig. 2) [5]. Additionally, the direct thrombin inhibitors reduce the platelet-aggregating effects of thrombin [14].

The effects of warfarin occur "upstream" from thrombin. Warfarin, a vitamin $\mathrm{K}$ antagonist, decreases the activity of
Fig. 1 Simplified diagram of the coagulation cascade demonstrating pharmacology of dabigatran
Intrinsic (contact activation) Pathway Extrinsic (tissue factor) Pathway

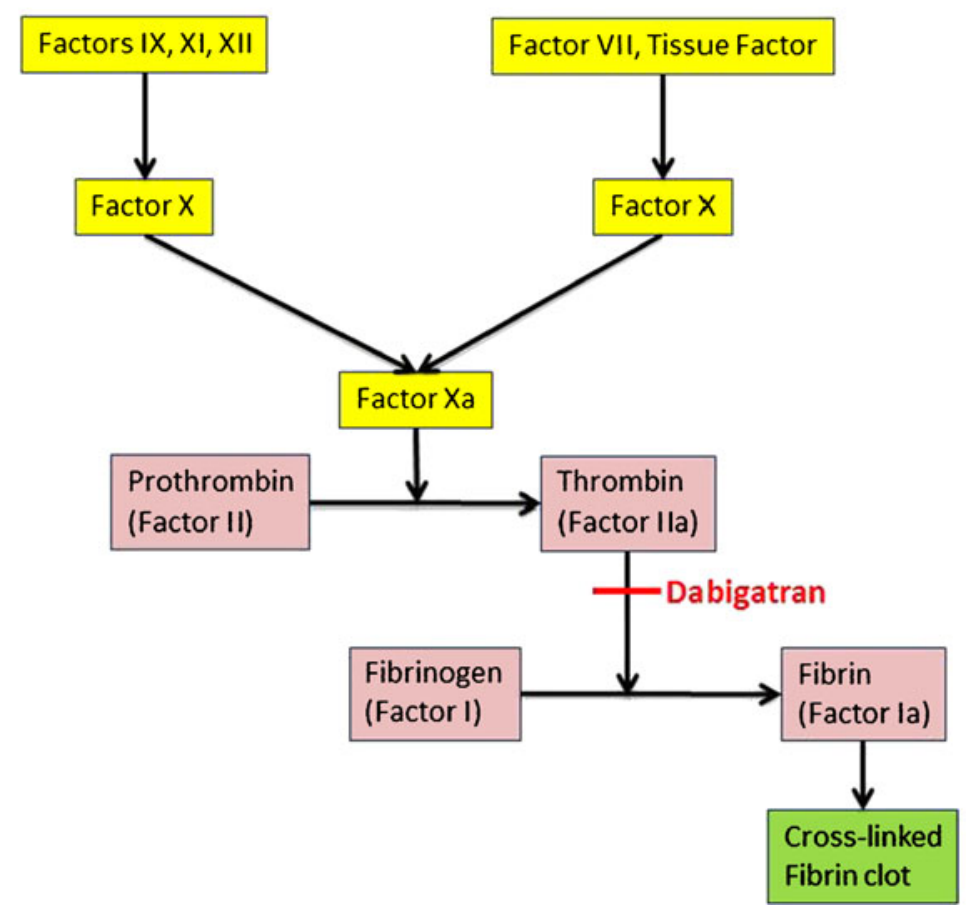




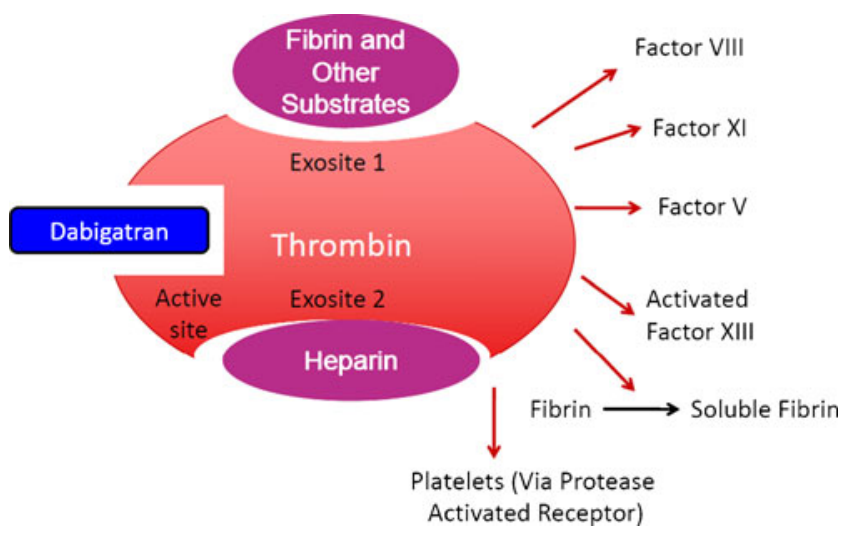

Fig. 2 Thrombin enables thrombus formation by activating multiple coagulation factors, catalyzing the conversion of fibrinogen to fibrin, and causing platelet aggregation. Dabigatran binds at the active site, thus inhibiting both free and fibrin-bound thrombin. Heparin exerts its action by increasing the affinity of antithrombin for thrombin. Heparin can only inhibit free thrombin since fibrin-bound thrombin has both exosites occupied and is relatively protected from the effects of antithrombin

the vitamin K-dependent clotting factors (II, VII, IX, and X), as well as proteins $\mathrm{C}$ and $\mathrm{S}$ [15]. Vitamin $\mathrm{K}$ absorption can be affected by diet and malabsorption (e.g., diarrhea), while warfarin, which is metabolized by CYP $1 \mathrm{~A} 2,3 \mathrm{~A} 4$, and $2 \mathrm{C} 9$, is susceptible to numerous food and drug interactions that can make anticoagulation with warfarin unpredictable [16, 17]. Also, genetic polymorphism in the CYP isoenzymes can create individual variation in dose response [18]. Dabigatran exhibits predictable and linear dose-dependent anticoagulation, leading to both the fixed dose regimen and lack of required hematologic monitoring in healthy volunteers [19].

\section{Pharmacokinetics}

Dabigatran is administered as the etexilate ester prodrug. Following absorption, the etexilate moiety is hydrolyzed by microsomal carboxylesterase to the active compound dabigatran, which produces immediate anticoagulation $[20,21]$. Its bioavailability is only $3-7 \%$ when the capsule is swallowed intact [22]. If the capsule shell is violated before ingestion, the oral bioavailability nearly doubles leading to manufacturer warnings that the capsules must not be cut, chewed, or opened prior to ingestion [23]. Peak plasma concentrations occur within $2 \mathrm{~h}$ of ingestion but may be delayed by concomitant food intake [23]. After the peak is reached, levels fall in a biphasic manner consistent with a rapid distribution phase and resulting in a more than $70 \%$ decrease within 4 to $6 \mathrm{~h}$ of ingestion [19, 20]. Approximately $35 \%$ of dabigatran is bound to plasma proteins and has a volume of distribution of 50 to $70 \mathrm{~L}[22,23]$.

Dabigatran is metabolized via conjugation into four acyl glucuronides, each of which is a direct thrombin inhibitor with less than $10 \%$ of the activity of the parent compound [23].
Dabigatran neither is metabolized by nor induces any cytochrome P450 subtype [22]. Dabigatran does, however, display P-glycoprotein (P-gp) efflux transporter-mediated interactions. For example, coadministration with rifampin (a P-gp inducer) decreases the peak dabigatran concentration by $67 \%$ [23]. While the area under the curve for the P-gp inhibitors verapamil and amiodarone increased in the presence of dabigatran, trough levels of both were unchanged [23]. Dabigatran has not been demonstrated to exert an effect on digoxin pharmacokinetics [24].

In patients with normal renal function, approximately $80 \%$ of an intravenous dabigatran dose is excreted in urine with an elimination half-life of $12-17 \mathrm{~h} \mathrm{[25].} \mathrm{Renal}$ impairment increases the elimination half-life to $15-34 \mathrm{~h}$ $[23,26]$. Dabigatran dosing therefore depends upon creatinine clearance (see Table 1). No dose adjustment is recommended for patients with hepatic impairment [27].

Dabigatran carries absolute contraindications in patients with acute bleeding or a history of serious hypersensitivity reaction to the drug itself [23]. Per the package insert, the risk of bleeding increases when dabigatran is administered with other anticoagulant and antiplatelet agents, such as warfarin, aspirin, and nonsteroidal anti-inflammatory medications [23]. However, an analysis of phase III trials where dabigatran was used for venothromboembolic event (VTE) prophylaxis in the setting of orthopedic surgery demonstrated no increase in major bleeding events during aspirin or NSAID coadministration [23]. Patients being transitioned from warfarin to direct thrombin inhibitors should have an international normalized ratio (INR) of less than 2.0 before initiation of dabigatran therapy [23].

Dabigatran is currently designated as pregnancy category $\mathrm{C}$ (no adequate and well-controlled studies in pregnant women) [23]. In rats, dabigatran exposure was associated with decreased implantation of fertilized ova, delayed ossification without major defects, an increased incidence of fetal demise, and maternal deaths at parturition due to hemorrhage [23]. The safety of dabigatran use in children and breastfeeding has not been established.

\section{Clinical Trials with Dabigatran}

Two large randomized trials have compared the safety and effectiveness of dabigatran against standard warfarin therapy.

Table 1 Dabigatran dosing adjustment based on creatinine clearance

\begin{tabular}{ll}
\hline Creatinine clearance & Dosing \\
\hline$>30 \mathrm{~mL} / \mathrm{min}$ & $150 \mathrm{mg}$ by mouth twice daily \\
$15-30 \mathrm{~mL} / \mathrm{min}$ & $75 \mathrm{mg}$ by mouth twice daily \\
$<15 \mathrm{~mL} / \mathrm{min}$ or hemodialysis & Not recommended \\
\hline
\end{tabular}


The RE-LY study compared dabigatran with warfarin for the prevention of embolic stroke in 18,113 patients with atrial fibrillation. Patients were randomized to receive warfarin, dabigatran $110 \mathrm{mg}$ twice daily, or dabigatran $150 \mathrm{mg}$ twice daily. The authors concluded that twice daily $110 \mathrm{mg}$ dabigatran doses produced rates of stroke and systemic emboli similar to the warfarin group $(1.53 \%$ vs $1.69 \%$, respectively) with a lower rate of major hemorrhage $(2.71 \%$ for dabigatran vs 3.36\% for warfarin). Doses of $150 \mathrm{mg}$ twice daily dabigatran resulted in a lower rate of stroke and systemic emboli compared to warfarin, but a similar rate of major hemorrhage. The rate of major bleeding in the higher dose dabigatran group was $3.11 \%$ per year and the rate of intracranial hemorrhage (ICH) was $0.10 \%$ per year compared to a rate of $3.36 \%$ of major hemorrhage and $0.38 \%$ of ICH in the warfarin group. Dabigatran also produced a dose-response improvement in mortality that was not statistically significant ( $4.13 \%$ per year for warfarin vs $3.75 \%$ per year for dabigatran $110 \mathrm{mg}$ vs $3.64 \%$ per year for dabigatran $150 \mathrm{mg}$ ) [28]. Of patients who had a major bleeding event associated with dabigatran, 57\% continued the medication after the event [29].

Subgroup analysis from the RE-LY trial revealed that older patients and those with chronic kidney disease were more likely to suffer bleeding complications. Patients over the age of 75 who received twice daily $150 \mathrm{mg}$ doses had a $5.1 \%$ per 100 patient-year rate of major bleeding. Patients with moderate renal impairment (creatinine clearance 30$50 \mathrm{~mL}$ per minute) who had dabigatran concentrations of two to three times that of patients with normal renal function had a $5.3 \%$ per 100 patient-year rate of major bleeding [29].

A second study (RE-COVER) compared dabigatran with warfarin for the treatment of acute VTE. Dabigatran given at a dose of $150 \mathrm{mg}$ twice daily produced no statistical difference in the incidence of recurrent VTE (2.1 vs $2.4 \%$ ) or major bleeding episodes (1.6 vs $1.9 \%$ ). The majority of bleeding events in the dabigatran group were either gastrointestinal or urogenital; no intracranial bleeding events in this group were recorded in this study [30].

Five randomized trials have compared dabigatran with enoxaparin for the prevention of VTE in patients undergoing elective orthopedic surgery [31-35]. Between 100 and $450 \mathrm{mg}$ total daily dose of dabigatran was compared to enoxaparin $30 \mathrm{mg}$ twice daily or $40 \mathrm{mg}$ daily. Most studies demonstrated noninferiority, while in one study, dabigatran fared worse [34] and in another, better at doses $300 \mathrm{mg} /$ day or greater [35]. Rates of major bleeding were similar in dabigatran (at daily doses of $220 \mathrm{mg}$ or less) and enoxaparin arms, with a single fatal retroperitoneal hemorrhage occurring in a patient receiving dabigatran [33]. Bleeding events tended to occur at the surgical sites. Increased daily doses of dabigatran of $300 \mathrm{mg}$ or greater increased the bleeding rate to $3.8-4.7 \%$ without fatalities [35].

\section{Monitoring of Anticoagulation}

The laboratory measurements that provide the most accurate assessment of the degree of anticoagulation are not readily available to most clinicians. For example, the thrombin time (TT) and ecarin clotting time (ECT) both demonstrate a linear response to serum dabigatran concentrations; the ECT provides the most accurate measurement of intensity of anticoagulation and is the most specific to direct thrombin inhibitors [19]. The TT assesses the activity of thrombin by measuring the time required to convert fibrinogen into fibrin in a plasma sample, thus measuring dabigatran activity. Commercial TT assays exist but have not entered widespread clinical use. Ecarin is a snake venom that converts prothrombin into meizothrombin, a metabolite that is inhibited by direct thrombin inhibitors, such as hirudin and dabigatran, but not by heparin. The ECT assay measures time to formation of clot in a plasma sample with added ecarin. Even though it is the most precise assay of intensity of anticoagulation, ECT is even less likely to be clinically available than TT. At this time, a thrombin time "like" clotting assay called the Hemoclot Thrombin Inhibitor kit, which is reported to have a direct correlation with serum dabigatran concentration and is useful for all direct thrombin inhibitors, is awaiting FDA approval [36].

Typical measures of clotting times have limited clinical utility in assessing the anticoagulant effect of dabigatran. The activated partial thromboplastin time (aPTT), a measure of the intrinsic (contact activation) pathway of the coagulation cascade to produce clot, does not respond linearly to the dose or intensity of anticoagulation with dabigatran. Instead, the aPTT reaches a plateau at higher doses and seems to peak at two to three times the control values. Thus, the aPTT in the setting of dabigatran therapy is merely a qualitative measure of effect and does not inform clinicians of the precise degree of anticoagulation. A normal aPTT would, however, exclude significant anticoagulation in a patient who takes dabigatran. The prothrombin time (PT) and the INR are also insensitive to serum dabigatran concentrations; although both these assays demonstrate linear responses, the slope of this response is fairly flat. Within therapeutic dabigatran serum concentrations, the INR would only be expected to be between 1.2 and 2.0 [19, 21, 37]. Just as with aPTT, both PT and INR are insufficiently precise to allow clinicians to judge the degree of anticoagulation.

\section{Management of Bleeding Complications}

Current recommendations for the management of dabigatranassociated hemorrhage are not based on evidence or clinical experience as this is such a new agent. For example, the 2011 $\mathrm{ACCF} / \mathrm{AHA}$ guidelines recommend that patients with severe 
dabigatran-associated hemorrhage be transfused with fresh frozen plasma (FFP) or packed red blood cells (pRBC); alternatively, surgical interventions to control hemorrhage may be used [1]. While transfusion of pRBC may support patients through a hemorrhagic episode and local control with direct pressure or sutures may stop superficial bleeding, the recommendation to transfuse FFP has only a questionable theoretical basis and as of yet, no clinical or animal evidence exists to support effectiveness. FFP contains prothrombin; transfusion would indirectly increase thrombin concentrations and possibly overcome dabigatran inhibition. However, most authors do not recommend using FFP since clotting factor inhibition is traditionally not expected to benefit significantly from plasma transfusion as would be the case for a factor depletion state [21, 38]. This argument is conceptually reasonable, especially in the setting of elevated serum dabigatran concentrations as would be expected to occur with overdose or renal failure.

The use of dabigatran presents new challenges for the management of acute, severe bleeding. While existing literature supports the safety of dabigatran over warfarin in large study populations, individuals receiving the drug will nonetheless suffer acute, life-threatening events, including bleeding in critical locations such as intracranial, spinal, and pericardial spaces. As of this writing, no agent has been accepted to effectively reverse the anticoagulation effects of dabigatran. As discussed above, FFP is of questionable theoretical benefit but carries only the mild risks associated with transfusion reactions. At the very least, transfusion of FFP will replace other factors that may be deficient (for example, if the patient is malnourished or has a dilutional coagulopathy) that could contribute to bleeding. Because thrombin acts independently of the factors found in cryoprecipitate, this agent would not be expected to accelerate clotting time.

Studies in rats suggest that recombinant-activated factor VII (rFVIIa) can reduce bleeding time and aPTT from high dose dabigatran. Even when tissue factor is not present, rFVIIa can generate thrombin [38]. However, human evidence supporting the benefit of rFVIIa administration on dabigatran-induced bleeding is mixed and must be inferred from experience with other direct thrombin inhibitors. Only one out of two healthy volunteer studies demonstrated that rFVIIa improved bleeding induced by melagatran; this is not an ideal comparison as melagatran has a larger volume of distribution compared to dabigatran [21]. In a case report, rFVIIa did slow lepirudin-induced bleeding after cardiac surgery [39].

Administration of activated prothrombin complex concentrates (APCC) has improved clotting times in both animal and human in vitro models of dabigatran-induced bleeding. Unfortunately, APCC are thrombogenic and their use can lead to ischemic events; in the absence of human clinical evidence, performing a risk-benefit analysis for the use of APCC to reverse dabigatran-associated coagulopathy is impossible. In our opinion, the strength of the current evidence is insufficient to advocate for transfusion of rFVIIa and APCC as a first-line therapy for dabigatran-induced bleeding. However, these agents should be considered as rescue therapy when there are signs of continuous and active bleeding that are life threatening, and local control fails or is not possible [21].

Dabigatran is renally excreted. Some accumulation of dabigatran is well tolerated; in the RE-LY trial, patients with moderate renal impairment had dabigatran serum concentrations of two to three times therapeutic and had modest increase in tendency to bleed (from $3.1 \%$ to $5.3 \%$ bleeding events per year). Patients maintained on dabigatran who develop acute renal insufficiency must, however, be treated with caution. Because serum dabigatran concentrations or degree of anticoagulation cannot be readily measured, treating clinicians should decrease dabigatran dosing or withhold the drug until renal insufficiency has resolved. Inpatient admission for patients at risk for falls should be considered along with measurement of serial hematocrits and frequent assessment of mental status, alterations in which may connote spontaneous bleeding. Additionally, adequate diuresis should be maintained in any patient with bleeding to promote renal elimination.

The dreaded complication of dabigatran is closed-space bleeding, whether intracranial, spinal, or pericardial. Although research data suggest these bleeding sites are less common for dabigatran than with warfarin, management of closed-space bleeding is more complicated because of the unavailability of reversal agents for dabigatran. Although a duration of bleeding that corresponds to dabigatran's elimination halflife of 12 to $17 \mathrm{~h}$ may not be lethal in gastrointestinal hemorrhage that can be treated with simple transfusion of pRBCs, protracted bleeding into the intracranial, spinal, or pericardial spaces would be devastating. We therefore recommend that emergent hemodialysis (HD) be considered as did van Ryn et al. [21]. The goal of HD would be to rapidly increase serum dabigatran elimination, thus limiting the extent of closed-space bleeding. Although published human experience is lacking, pharmacokinetic evidence suggests that HD would provide significant benefit since the degree of anticoagulation is correlated with serum concentrations, particularly in patients with decreased renal function. In a pharmacokinetic analysis of nonbleeding volunteers with end-stage renal disease, HD removes $62 \%$

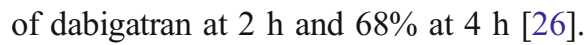

HD would be expected to create fluid shifts and potentially decrease plasma osmolarity which would increase cerebral water content, an undesirable sequela in a patient with an ICH. Preventive measures, including the administration of intravenous mannitol or a high-sodium dialysate to increase plasma 
osmolarity, can be considered in consultation with the treating nephrologist [40]. Abnormalities in blood pressures should be aggressively controlled to maintain cerebral perfusion. Because patients receiving HD may develop systemic hypotension leading to cerebral hypoperfusion, low blood pressure should be aggressively supported through fluid resuscitation or, potentially, vasoactive amine infusions. Careful placement of a central venous catheter for dialysis and avoidance of any added anticoagulant to the dialysis circuit would be important so as not to risk increased bleeding. Finally, patients with dabigatran-induced intracranial hemorrhage deserve frequent neurologic examinations and repeat head CT if mental status deteriorates.

The inability to readily and accurately assess the degree of anticoagulation has broad implications for surgical intervention. Assays that accurately measure the degree of anticoagulation in dabigatran therapy-thrombin time, ecarin clotting time, and, potentially, dabigatran serum concentrations - are unavailable to most clinicians. Even though a normal aPTT will exclude an anticoagulated state, an elevated aPTT only provides qualitative evidence that increased risk of bleeding exists. Until these assays become more commonly available, clinicians can predict only with difficulty extent and duration of dabigatran-induced bleeding episodes. Additionally, clinicians confronted by a surgical emergency (i.e., perforated abdominal viscus) in a patient with a prolonged aPTT from dabigatran can anticipate neither the extent of procedural blood loss nor the time needed to reverse anticoagulation. Clinicians should carefully weigh the risk of emergent surgery with increased bleeding against the risk of nonoperative care and waiting until aPTT has normalized. As with dabigatranassociated bleeding, no sound human evidence suggests transfusion of blood products hastens reversal of anticoagulation or decreases intraoperative bleeding.

Finally, no clinical experience with dabigatran overdose exists. In experimental models, charcoal has been shown to adsorb dabigatran [21]. Since two- to threefold increases in serum concentration are well tolerated, a patient who has an accidental ingestion or therapeutic misadventure of dabigatran would not be expected to have bleeding complications. Conversely, a patient with a large intentional ingestion could develop bleeding complications. If bleeding occurs, and the extent of bleeding is severe, HD should be considered to rapidly increase elimination as suggested for closed-space bleeding.

\section{Summary}

Dabigatran is a novel oral direct thrombin inhibitor recently approved by the FDA for stroke prevention in patients with nonvalvular atrial fibrillation. Since frequent monitoring of degree of anticoagulation is unnecessary and food and drug interactions are minimal, dabigatran offers significant benefit and ease of use compared to warfarin. There is increasing demand for dabigatran among patients who take warfarin, or who have been unable to tolerate warfarin, and this is only expected to increase. Clinical trials suggest comparable or lesser rates of major hemorrhage compared to warfarin. Unlike warfarin, anticoagulation from dabigatran cannot be readily reversed with current interventions. The risk of dabigatran-induced bleeding may potentially worsen in patients with acute or chronic renal impairment. New challenges are expected to arise with increased use of dabigatran, such as assessing degree of anticoagulation, management of closed-space bleeding, and referring patients who may need emergent surgery. Management of bleeding complications should start with local control if possible and transfusion of pRBCs if needed. Transfusion of FFP is generally considered ineffective, while rFVIIa and APCC should be considered as rescue therapy for active bleeding even though data are limited and potential exists for ischemic complications. Hemodialysis will increase elimination of dabigatran and should be considered as another potential rescue therapy, especially if renal failure is present. Further study is needed of dabigatran-induced bleeding complications, their extent and management, and risks and benefit of transfusion of blood products versus hemodialysis.

\section{References}

1. Wann LS, Curtis AB, Ellenbogen KA et al (2011) 2011 ACCF/ AHA/HRS focused update on the management of patients with atrial fibrillation (update on dabigatran): a report of the American College of Cardiology Foundation/American Heart Association Task Force on practice guidelines. J Am Coll Cardiol 57 (11):1330-1337

2. Melnikova I (2009) The anticoagulants market. Nat Rev Drug Discov 8(5):353-354

3. Hauel NH, Nar H, Priepke H, Ries U, Stassen JM, Wienen W (2002) Structure-based design of novel potent nonpeptide thrombin inhibitors. J Med Chem 45(9):1757-1766

4. Wienen W, Stassen JM, Priepke H, Ries UJ, Hauel N (2007) Invitro profile and ex-vivo anticoagulant activity of the direct thrombin inhibitor dabigatran and its orally active prodrug, dabigatran etexilate. Thromb Haemost 98(1):155-162

5. Di Nisio M, Middeldorp S, Buller HR (2005) Direct thrombin inhibitors. N Engl J Med 353(10):1028-1040

6. Coughlin SR (2000) Thrombin signalling and protease-activated receptors. Nature 407(6801):258-264

7. Bar-Shavit R, Hruska KA, Kahn AJ, Wilner GD (1987) Thrombin chemotactic stimulation of HL-60 cells: studies on thrombin responsiveness as a function of differentiation. J Cell Physiol 131 (2):255-261

8. Griffith MJ (1982) Kinetics of the heparin-enhanced antithrombin $\mathrm{III} /$ thrombin reaction. Evidence for a template model for the mechanism of action of heparin. J Biol Chem 257(13):7360-7365

9. Jordan RE, Oosta GM, Gardner WT, Rosenberg RD (1980) The kinetics of hemostatic enzyme-antithrombin interactions in the 
presence of low molecular weight heparin. J Biol Chem 255 (21):10081-10090

10. Tulinsky A (1996) Molecular interactions of thrombin. Semin Thromb Hemost 22(2):117-124

11. Liaw PC, Becker DL, Stafford AR, Fredenburgh JC, Weitz JI (2001) Molecular basis for the susceptibility of fibrin-bound thrombin to inactivation by heparin cofactor II in the presence of dermatan sulfate but not heparin. J Biol Chem 276(24):20959-20965

12. Weitz JI, Hudoba M, Massel D, Maraganore J, Hirsh J (1990) Clot-bound thrombin is protected from inhibition by heparinantithrombin III but is susceptible to inactivation by antithrombin III-independent inhibitors. J Clin Invest 86(2):385-391

13. Weitz JI, Leslie B, Hudoba M (1998) Thrombin binds to soluble fibrin degradation products where it is protected from inhibition by heparin-antithrombin but susceptible to inactivation by antithrombin-independent inhibitors. Circulation 97(6):544-552

14. Xiao Z, Theroux P (1998) Platelet activation with unfractionated heparin at therapeutic concentrations and comparisons with a lowmolecular-weight heparin and with a direct thrombin inhibitor. Circulation 97(3):251-256

15. Freedman MD (1992) Oral anticoagulants: pharmacodynamics, clinical indications and adverse effects. J Clin Pharmacol 32 (3):196-209

16. Wells PS, Holbrook AM, Crowther NR, Hirsh J (1994) Interactions of warfarin with drugs and food. Ann Intern Med 121(9):676-683

17. Arnason T, Wells PS, van Walraven C, Forster AJ (2006) Accuracy of coding for possible warfarin complications in hospital discharge abstracts. Thromb Res 118(2):253-262

18. Schwarz UI, Ritchie MD, Bradford Y et al (2008) Genetic determinants of response to warfarin during initial anticoagulation. N Engl J Med 358(10):999-1008

19. Stangier J, Rathgen K, Stahle H, Gansser D, Roth W (2007) The pharmacokinetics, pharmacodynamics and tolerability of dabigatran etexilate, a new oral direct thrombin inhibitor, in healthy male subjects. Br J Clin Pharmacol 64(3):292-303

20. Stangier J (2008) Clinical pharmacokinetics and pharmacodynamics of the oral direct thrombin inhibitor dabigatran etexilate. Clin Pharmacokinet 47(5):285-295

21. van Ryn J, Stangier J, Haertter S et al (2011) Dabigatran etexilate - a novel, reversible, oral direct thrombin inhibitor: interpretation of coagulation assays and reversal of anticoagulant activity. Thromb Haemost 103(6): 1116-1127

22. Blech S, Ebner T, Ludwig-Schwellinger E, Stangier J, Roth W (2008) The metabolism and disposition of the oral direct thrombin inhibitor, dabigatran, in humans. Drug Metab Dispos 36(2):386-399

23. Pradaxa [package insert]. Boehringer Ingelheim Pharmaceuticals, Inc, Ridgefield, CT; March 2011. http://www.accessdata.fda.gov/ drugsatfda_docs/label/2011/022512s004lbl.pdf. Accessed 4 Aug 2011

24. Stangier J, Stahle H, Rathgen K. No interaction of the oral direct thrombin dabigatran etexilate and digoxin abstract no. P-W-672; online. Available from URL: http://isth2007.abstractsondemand.com/ Accessed 30 June 2011

25. Stangier J, Stahle H, Rathgen K, Fuhr R (2008) Pharmacokinetics and pharmacodynamics of the direct oral thrombin inhibitor dabigatran in healthy elderly subjects. Clin Pharmacokinet 47 (1):47-59

26. Stangier J, Rathgen K, Stahle H, Mazur D (2011) Influence of renal impairment on the pharmacokinetics and pharmacodynamics of oral dabigatran etexilate: an open-label, parallel-group, singlecentre study. Clin Pharmacokinet 49(4):259-268

27. Stangier J, Stahle H, Rathgen K, Roth W, Shakeri-Nejad K (2008) Pharmacokinetics and pharmacodynamics of dabigatran etexilate, an oral direct thrombin inhibitor, are not affected by moderate hepatic impairment. J Clin Pharmacol 48(12):1411-1419

28. Connolly SJ, Ezekowitz MD, Yusuf S et al (2009) Dabigatran versus warfarin in patients with atrial fibrillation. N Engl J Med 361(12):1139-1151

29. Beasley BN, Unger EF, Temple R (2011) Anticoagulant optionswhy the FDA approved a higher but not a lower dose of dabigatran. N Engl J Med 364(19):1788-1790

30. Schulman S, Kearon C, Kakkar AK et al (2009) Dabigatran versus warfarin in the treatment of acute venous thromboembolism. $\mathrm{N}$ Engl J Med 361(24):2342-2352

31. Eriksson BI, Dahl OE, Huo MH et al (2011) Oral dabigatran versus enoxaparin for thromboprophylaxis after primary total hip arthroplasty (RE-NOVATE II*). A randomised, double-blind, noninferiority trial. Thromb Haemost 105(4):721-729

32. Eriksson BI, Dahl OE, Rosencher N et al (2007) Oral dabigatran etexilate vs. subcutaneous enoxaparin for the prevention of venous thromboembolism after total knee replacement: the RE-MODEL randomized trial. J Thromb Haemost 5(11):2178-2185

33. Eriksson BI, Dahl OE, Rosencher N et al (2007) Dabigatran etexilate versus enoxaparin for prevention of venous thromboembolism after total hip replacement: a randomised, double-blind, non-inferiority trial. Lancet 370(9591):949-956

34. The RE-MOBILIZE Writing Committee, Ginsberg JS, Davidson BL et al (2009) Oral thrombin inhibitor dabigatran etexilate vs North American enoxaparin regimen for prevention of venous thromboembolism after knee arthroplasty surgery. J Arthroplasty 24(1):1-9

35. Eriksson BI, Dahl OE, Buller HR et al (2005) A new oral direct thrombin inhibitor, dabigatran etexilate, compared with enoxaparin for prevention of thromboembolic events following total hip or knee replacement: the BISTRO II randomized trial. J Thromb Haemost 3 (1):103-111

36. Aniara. http://dabigatrantesting.com/. Accessed 17 May 2011

37. Freyburger G, Macouillard G, Labrouche S, Sztark F (2011) Coagulation parameters in patients receiving dabigatran etexilate or rivaroxaban: two observational studies in patients undergoing total hip or total knee replacement. Thromb Res 127(5):457-465

38. Crowther MA, Warkentin TE (2009) Managing bleeding in anticoagulated patients with a focus on novel therapeutic agents. J Thromb Haemost 7(Suppl 1):107-110

39. Oh JJ, Akers WS, Lewis D, Ramaiah C, Flynn JD (2006) Recombinant factor VIIa for refractory bleeding after cardiac surgery secondary to anticoagulation with the direct thrombin inhibitor lepirudin. Pharmacotherapy 26(4):569-577

40. Davenport A (2008) Practical guidance for dialyzing a hemodialysis patient following acute brain injury. Hemodial Int 12(3):307-312 